Yslado, R., Ramírez-Asís, E., García-Figueroa, M. \& Arquero, J. (2021). Clima laboral y burnout en profesores universitarios. Revista Electrónica Interuniversitaria de Formación del Profesorado, 24(3), 101-114.

DOI: https://doi.org/10.6018/reifop.476651

\title{
Clima laboral y burnout en profesores universitarios
}

Rosario Yslado ${ }^{(1)}$, Edwin Ramírez-Asís ${ }^{(1)}$, María García-Figueroa ${ }^{(1)}$, José Arquero ${ }^{(2)}$

(1) Universidad Nacional Santiago Antúnez de Mayolo, Perú. ${ }^{(2)}$ Universidad de Sevilla, España.

\section{Resumen}

El objetivo de la presente investigación fue conocer la relación funcional entre el clima laboral y el síndrome de burnout en profesores universitarios de una universidad pública de Perú. La muestra estuvo constituida por 206 profesores; nombrados (80.1\%) y contratados (19.9\%); varones (79.6\%) y mujeres (20.4\%). Los instrumentos utilizados fueron: el Cuestionario de burnout para docentes universitarios adaptado por Arquero y Donoso a partir del Inventario de Burnout de Maslach y la Escala de Clima Laboral de Palma. Se utilizó el análisis factorial confirmatorio para verificar la validez y fiabilidad. Se encontró que el nivel del clima laboral fue promedio ( $\bar{x}=138.8$ puntos); los profesores presentaron burnout de nivel medio $\left(x^{-}=115.8\right.$ puntos). A través del modelo de ecuaciones estructurales, se encontró que el factor supervisión del clima laboral está relacionado con la dimensión de despersonalización del burnout. Además, realizar varios trabajos remunerados genera mayor nivel de burnout $(p=0.040)$. Se concluye que existe relación negativa y estadísticamente significativa entre el clima laboral y el burnout. Asimismo, clima laboral predice los niveles de síndrome de burnout $(A G F I=0.991)$ en los profesores universitarios. El estudio sugiere la implementación de programas de intervención ocupacional y organizacional.

\section{Palabras clave}

Clima laboral; burnout; modelo de ecuaciones estructurales; profesores universitarios.

\section{Work climate and burnout in university professor}

\section{Abstract}

The objective of this research was to know the functional relationship between the work environment and the burnout syndrome in university professors from a public university in Peru. The sample consisted of 206 teachers; males (79.6\%) and females (20.4\%); appointed (80.1\%) and hired (19.9\%). The instruments used were: The Burnout Questionnaire for 
university teachers adapted by Arquero and Donoso from the Maslach Burnout Inventory and the Palma Work Climate Scale. Confirmatory factor analysis was used to verify validity and reliability. It was found that the level of the work environment was average $\left(x^{-}=138.8\right.$ points); the teachers presented medium level burnout ( $x^{-}=115.8$ points). Through the structural equations model, it was found that the supervisory factor of the work environment is related to the depersonalization dimension of burnout. Likewise, performing various paid jobs generates a higher level of burnout $(p=0.040)$. It is concluded that there is a negative and statistically significant relationship between the work environment and burnout. Likewise, the work environment predicts the levels of burnout syndrome (AGFI $=0.991$ ) in university professors. The study suggests the implementation of occupational and organizational intervention programs.

\section{Key words}

Working environment; burnout; structural equation model; University professor.

\section{Introducción}

Se define el clima laboral (CL) como la percepción de las características de la organización, perdurables y medibles, que distinguen una entidad laboral de otra y permite hacer un diagnóstico, que orienta acciones preventivas y correctivas necesarias para optimizar y/o fortalecer el funcionamiento de procesos y resultados organizacionales (Palma, 2004). La medición periódica del clima laboral es importante porque realimenta a las universidades públicas, para mejorar no solamente el ambiente de trabajo, sino la calidad de vida laboral y de los servicios educativos e investigación, que se realizan en los entornos de universidades públicas (Hernández, Garrido y Rico, 2016; Ramírez et al., 2020).

De otra parte, el síndrome de burnout (SB) es una respuesta al estrés laboral crónico, caracterizado por el agotamiento físico y emocional, despersonalización y la reducción de la capacidad personal de logro (Ilaja y Reyes, 2016), y al respecto se han realizado muchas investigaciones, que afirman el incremento del burnout en los profesores universitarios (Rodríguez-García, Sola-Martínez y Fernández-Cruz, 2017); el burnout puede ocasionar la suspensión temporal o permanente del ejercicio de la profesión docente (Usán, Salavera y Domper, 2018).

Las universidades desempeñan un rol importante en el desarrollo económico, político, social y cultural de los países. La sociedad exige y responsabiliza socialmente a la universidad y sus profesores a formar talentos humanos en diferentes disciplinas profesionales, que sean competitivos para que puedan enfrentar los cambios del mundo globalizado, las demandas del mercado laboral y la revolución tecnológica creciente; y que sean profesionales a la vanguardia en la producción de investigaciones. Para que las universidades cumplan exitosamente los roles que les asigna la sociedad y el mercado ocupacional, necesitan atraer y mantener una plana de profesores de calidad, a quienes deben apoyar y proveer de un clima de trabajo favorable (Rodríguez-García et al., 2017).

Los estudios internacionales y nacionales precedentes confirman que los profesores de educación básica y de educación superior no universitaria presentan diferentes niveles de burnout (Abkhou y Jenaabadi, 2015; Arís, 2017; De Araujo et al., 2019; Espinoza, Tous-Pallarès y Vigil-Colet, 2015). Para el caso de los profesores universitarios, el burnout se está incrementando en el presente siglo e implica un alto costo para la administración educativa (Arquero y Donoso, 2013; Ilaja y Reyes, 2016; Morales et al., 2016; Ruíz de Chávez et al., 2014; Cárdenas, Méndez y González, 2014; Yslado, Ramírez y Espinoza, 2020). 
Los profesores nombrados y contratados de las universidades públicas, en su mayoría, además de cumplir con la función de docencia y responsabilidad social, realizan investigaciones y deben responder a las exigencias propias de la universidad y de sus usuarios. También afrontan los estresores percibidos en el contexto de trabajo: los fondos y recursos insuficientes, dificultades financieras, sobrecarga de trabajo, malas prácticas de gestión, inseguridad laboral, reconocimiento y recompensa insuficiente, condiciones inadecuadas de trabajo y aspectos estructurales de la tarea docente y la interacción problemática con los estudiantes (Rodríguez-García et al., 2017; Lou y Chen, 2016). Todos estos aspectos les generan desgaste profesional (burnout) que afectan tanto su desempeño laboral, salud integral y bienestar personal, como la calidad de los servicios educativos, que es exigido para las universidades del contexto internacional y específicamente para el caso de Perú lo requiere el Sistema Nacional de Evaluación, Acreditación y Certificación de la Calidad Educativa de Perú (SINEACE, 2017).

En el Perú existe pocos estudios sobre burnout en profesores universitarios, quienes constituyen el capital humano de las universidades. Los estudios precedentes en su mayoría son cuantitativos y descriptivos (analizan la frecuencia, las características de las dimensiones del burnout), y otros son correlacionales, entre otros estudian los factores sociodemográficos y desempeño docente asociados al burnout (Carpio y Requis, 2016).

Al revisar la literatura, se encontraron implicaciones teóricas sobre la relación entre el clima laboral y el burnout (Esquer et al., 2019; Palma, 2004). Asimismo, se encontraron pocos estudios sobre el burnout en muestras de profesores universitarios (Usán et al., 2018); sin embargo no se han encontrado investigaciones sobre la relación entre el $\mathrm{CL}$ y el SB en profesores universitarios, establecida a través del modelo de ecuaciones estructurales (SEM, por sus siglas en inglés), lo cual se llevó a cabo en el presente estudio para completar este vacío, que permitió conocer un panorama más amplio del comportamiento del burnout, relacionado al $\mathrm{CL}$ en un ámbito de Perú, e informa datos estadísticos útiles para una mejor comprensión del problema estudiado y proponer programas de intervención ocupacional y organizacional.

En línea con lo expuesto, el objetivo general del presente estudio fue: Conocer la relación funcional entre el clima laboral y el síndrome de burnout en los profesores de una universidad pública de Perú; y el objetivo específico fue establecer comparación entre los niveles del clima laboral y del burnout, según variables sociodemográficas-laborales.

\section{Metodología}

Diseño del estudio: Es un estudio cuantitativo-correlacional, no experimental y transversal, realizado en profesores de una universidad pública de Ancash (Perú), que es multidisciplinaria, cuenta con 11 facultades (Ciencias; Ciencias Agrarias; Ingeniería de industrias alimentarias; Ingeniería civil; Ingeniería de mina; Ciencias del ambiente; Ciencias médicas; Economía y contabilidad; Administración y turismo; Derecho y ciencias políticas; Ciencias sociales, educación y comunicación), y 24 carreras profesionales.

Población y muestra: La población de estudio fue de 422 profesores de la universidad referida. El muestreo fue aleatorio, estratificado con afijación proporcional por facultades, con un error de $5 \%$ y un nivel de confianza de 95\% (Hernández y Carpio, 2019). Participaron un total de 206 profesores de todas las facultades citadas, cuyas edades oscilaron entre 30 a 70 años de edad, el $79.6 \%$ fueron varones; el $71.4 \%$ fueron casados; el $80.1 \%$ nombrados; $35.9 \%$ con experiencia laboral de 21 hasta 30 años; el 80.6\%, tenía solo un trabajo remunerado y la mayoría (36.9\%) trabajaba cuatro horas diarias en contacto con los estudiantes. 
Técnica e instrumentos: Se utilizó la encuesta y se aplicaron los siguientes instrumentos:

a) Ficha sociodemográfica: Incluye 8 ítems referidos al sexo, edad, estado civil, condición laboral, tiempo de experiencia laboral, número de trabajos remunerados, número de horas semanales laboradas y número de horas de contacto directo con los estudiantes de la UNASAM.

b) Escala de clima laboral Cl-SPC (Palma, 2004): Consta de 50 ítems cuyas normas interpretativas (baremos) se han obtenido en una muestra peruana. Previo a su aplicación se determinó sus propiedades psicométricas, que fueron adecuadas (Tabla 1).

Tabla 1.

Propiedades psicométricas de la Escala de Clima Laboral-SPC

\begin{tabular}{|c|c|c|c|c|c|c|c|}
\hline \multicolumn{2}{|c|}{ Características } & $\begin{array}{l}\text { Clima } \\
\text { laboral }\end{array}$ & $\begin{array}{l}\text { Realización } \\
\text { Personal }\end{array}$ & $\begin{array}{c}\text { Involucramiento } \\
\text { Laboral }\end{array}$ & Supervisión & Comunicación & $\begin{array}{c}\text { Condiciones } \\
\text { Laborales }\end{array}$ \\
\hline \multicolumn{2}{|c|}{ Número de ítems } & 50 & 10 & 10 & 10 & 10 & 10 \\
\hline \multicolumn{2}{|c|}{ Ítems que la conforman } & $1-50$ & $\begin{array}{l}1,6,11,16 \\
21,26,31 \\
36,41,46\end{array}$ & $\begin{array}{c}2,7,12,17,22, \\
27,32,37,42,47\end{array}$ & $\begin{array}{l}3,8,13,18, \\
23,28,33, \\
38,43,48\end{array}$ & $\begin{array}{c}4,9,14,19 \\
24,29,34,39 \\
44,49\end{array}$ & $\begin{array}{c}5,10,15,20 \\
25,30,35 \\
40,45,50\end{array}$ \\
\hline \multirow{2}{*}{$\begin{array}{c}\text { Puntaje } \\
\text { (muestral) }\end{array}$} & Mínimo & 74 & 12 & 16 & 12 & 13 & 14 \\
\hline & Máximo & 246 & 50 & 50 & 49 & 49 & 48 \\
\hline \multirow{2}{*}{\multicolumn{2}{|c|}{$\begin{array}{c}\text { Fiabilidad (Alfa de } \\
\text { Cronbach) } \\
\text { Validez de contenido } \\
\text { (Lawshe) }\end{array}$}} & 0.964 & 0.881 & 0.813 & 0.861 & 0.884 & 0.834 \\
\hline & & 1.000 & 1.000 & 1.000 & 1.000 & 1.000 & 1.000 \\
\hline \multirow{3}{*}{ Percentil } & P25 & 124.75 & 22.00 & 29.00 & 25.00 & 22.00 & 24.00 \\
\hline & Mediana & 138.00 & 25.00 & 32.50 & 28.00 & 25.00 & 27.00 \\
\hline & P75 & 155.00 & 29.25 & 36.00 & 32.00 & 29.00 & 30.00 \\
\hline \multicolumn{2}{|c|}{ Media } & 138.85 & $25 \cdot 50$ & 32.20 & 28.33 & 25.73 & 27.09 \\
\hline \multicolumn{2}{|c|}{ Desviación estándar } & 26.726 & 6.163 & $5 \cdot 512$ & 6.140 & 6.002 & $5 \cdot 540$ \\
\hline \multirow{5}{*}{$\begin{array}{l}\text { Puntos de } \\
\text { corte*** } \\
(T=50+ \\
10 * Z)\end{array}$} & $\begin{array}{l}\text { Muy } \\
\text { insatisfecho }\end{array}$ & $\begin{array}{c}74 a \\
122\end{array}$ & 12 a 21 & 16 a 28 & 12 a 24 & 13 a 21 & 14 a 23 \\
\hline & Insatisfecho & $\begin{array}{c}123 a \\
134\end{array}$ & 22 a 24 & 29 a 31 & 25 a 27 & 22 a 24 & 24 a 26 \\
\hline & Promedio & $\begin{array}{c}135 \mathrm{a} \\
147\end{array}$ & 25 a 27 & 32 a 34 & 28 a 30 & 25 a 27 & 27 a 28 \\
\hline & Satisfecho & $\begin{array}{c}148 \mathrm{a} \\
159\end{array}$ & 28 a 30 & 35 a 36 & 31 a 32 & 28 a 30 & 29 a 31 \\
\hline & $\begin{array}{l}\text { Muy } \\
\text { satisfecho }\end{array}$ & $\begin{array}{l}160 a \\
246\end{array}$ & 31 a 50 & 37 a 50 & 33 a 49 & 31 a 49 & 32 a 48 \\
\hline
\end{tabular}

Nota: a/ Los puntos de corte son cada $20 \%$

c) Cuestionario de burnout para profesores universitarios: Es una versión modificada (Arquero y Donoso, 2006), y se obtuvo a partir de la adaptación española del MBI de Maslach y Jackson (1997). Asimismo, se determinó sus propiedades psicométricas en el contexto de investigaciones y estas fueron adecuadas (Tabla 2). 
Tabla 2.

Propiedades psicométricas del Cuestionario Burnout para profesores universitarios

\begin{tabular}{|c|c|c|c|c|c|}
\hline \multicolumn{2}{|c|}{ Características } & $\begin{array}{l}\text { Síndrome de } \\
\text { Burnout (SB) }\end{array}$ & $\begin{array}{c}\text { Cansancio } \\
\text { emocional (CE) }\end{array}$ & $\begin{array}{l}\text { Despersonali- } \\
\text { zación (DP) }\end{array}$ & $\begin{array}{c}\text { Realización } \\
\text { personal (RP) }\end{array}$ \\
\hline \multicolumn{2}{|l|}{ Número de ítems } & 34 & 14 & 6 & 14 \\
\hline \multicolumn{2}{|c|}{ Ítems que la conforman } & $1-34$ & $\begin{array}{c}1,2,3,4,7,8,11 \\
12,19,20,21 \\
24,25,32\end{array}$ & $\begin{array}{c}6,15,16,22,23 \\
34\end{array}$ & $\begin{array}{c}5,9,10,13,14 \\
17,18,26,27 \\
28,29,30,31 \\
33\end{array}$ \\
\hline \multirow{2}{*}{$\begin{array}{l}\text { Puntaje } \\
\text { (muestral) }\end{array}$} & Mínimo & 60 & 14 & 6 & 37 \\
\hline & Máximo & 151 & 62 & 26 & 84 \\
\hline \multicolumn{2}{|c|}{ Fiabilidad (Alfa de Cronbach) } & 0.687 & 0.899 & 0.632 & 0.874 \\
\hline \multicolumn{2}{|c|}{ Validez de contenido (Lawshe) } & 1.000 & 1.000 & 1.000 & 1.000 \\
\hline \multirow{3}{*}{ Percentil } & $\mathrm{P} 25$ & 107.00 & 25.00 & 8.00 & 64.00 \\
\hline & Mediana & $114 \cdot 50$ & 33.00 & 11.00 & 71.00 \\
\hline & P75 & 123.00 & 43.00 & 16.00 & 76.25 \\
\hline \multirow{2}{*}{\multicolumn{2}{|c|}{$\begin{array}{l}\text { Media } \\
\text { Desviación estándar }\end{array}$}} & 115.77 & 34.30 & 11.98 & 69.50 \\
\hline & & 12.309 & 11.960 & 4.885 & 9.095 \\
\hline \multirow{3}{*}{$\begin{array}{l}\text { Puntos de corte } * * * \\
\left(T=50+10^{*} Z\right)\end{array}$} & Baja & 60 a 110 & 14 a 28 & 6 a 9 & 75 a 84 \\
\hline & Media & 111 a 120 & 29 a 39 & 10 a 14 & 68 a 74 \\
\hline & Alta & 121 a 151 & 40 a 62 & 15 a 26 & 37 a 67 \\
\hline
\end{tabular}

Nota: a/ Los puntos de corte son cada 33\%

\section{Procedimiento}

Inicialmente los vicerrectores, académico y de investigación de la UNASAM autorizaron la solicitud para la recolección de los datos. Seguidamente se procedió a verificar las propiedades psicométricas de los instrumentos de investigación y se capacitó a los jefes de encuesta y encuestadores. El Comité de ética de la UNASAM evaluó y aprobó el proyecto de investigación. Adicionalmente, se consideraron los principios éticos establecidos en la Declaración de Helsinki (Mazzanti, 2011); es decir, los instrumentos de investigación se administraron de forma anónima y previa aceptación del consentimiento informado escrito de los participantes. La recolección de datos tuvo lugar durante el mes de abril del 2019.

\section{Procesamiento de datos}

Se realizó el análisis del modelo de medición, que consistió en relacionar la variable CL con la variable SB, por medio de la estimación del modelo de ecuaciones estructurales, las variables latentes se conformaron con las variables observadas (Collier, 2020). Se realizó el análisis mediante el método de estimación de distribución libre asintótica, ya que las variables analizadas no cumplieron el supuesto de normalidad multivariante $\left(K^{\wedge} 2=19.172\right.$; g.l. $=2$; $p<0.001)$. En cuanto a la valoración del ajuste del modelo, se usó el Chi-Cuadrado $\left(\chi^{\wedge} 2\right.$, $p \geq 0.05$ ), la Aproximación de la Raíz de Cuadrados Medios del Error (RMSEA $\leq 0.05$ ) y el Índice de Ajuste Comparativo (CFI>0.95) (Collier, 2020).

Además, se llevó a cabo el análisis del modelo estructural, que se refiere a las relaciones entre las variables latentes o factores formados, y entre estos y las variables observadas. En este estudio se hipotetizó que los factores del CL afectarían las dimensiones de SB. Se propuso un modelo de ecuaciones estructurales (Figura 1), a fin de analizar la relación funcional entre el $\mathrm{CL}$ (variable exógena) sobre el SB (variable endógena). Se siguieron las etapas propuestas por Medrano y Muñoz-Navarro (2017), partiendo con la especificación del modelo de medición. 
Figura 1.

Modelo estructural hipotetizado.

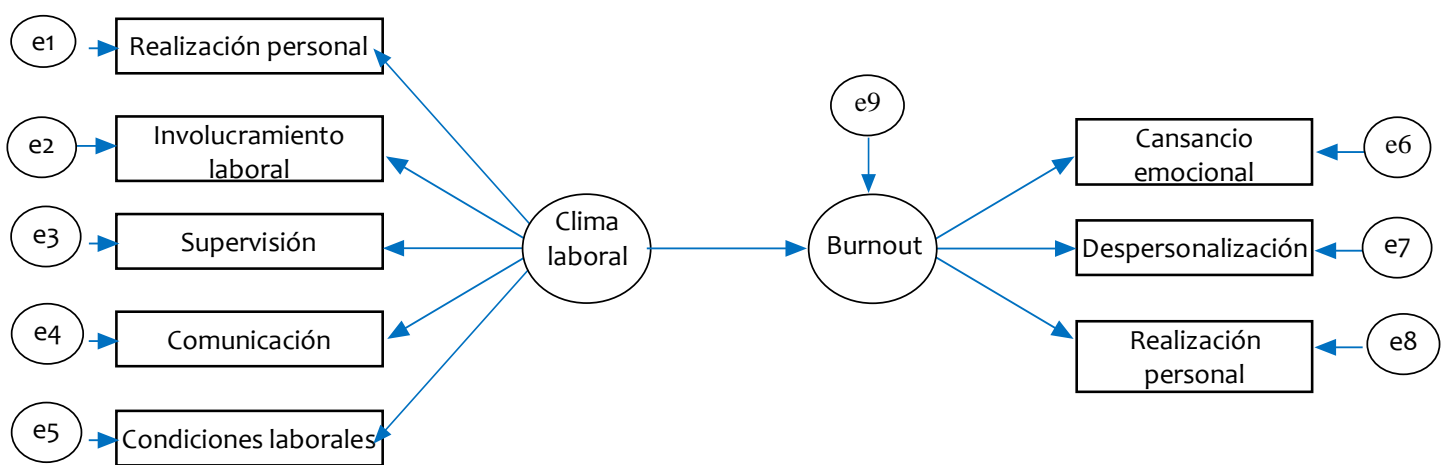

Tras la identificación del modelo, se realizó la estimación de los coeficientes de las relaciones lineales y las relaciones de covariabilidad (Medrano y Muñoz-Navarro, 2017). Respecto a la reespecificación del modelo, se introdujeron parámetros adicionales que favorecieron la explicación de la relación entre las variables. El modelo propuesto fue valorado estadísticamente en función a la suficiencia de los índices de ajuste $\left(\chi^{\wedge} 2, p=.998 \geq 0.05\right.$; RMSEA $=.000 \leq 0.05 ; \mathrm{CFI}=1.000>.95)$.

Adicionalmente se compararon los niveles del $\mathrm{CL}$ y del $\mathrm{SB}$, según las variables sociodemográficas-laborales de la muestra (sexo, edad, estado civil, condición laboral, tiempo de experiencia laboral, número de trabajos remunerados y horas laboradas diariamente). Respecto al $\mathrm{CL}$, la medición se realizó con la inferencia paramétrica: prueba $\mathrm{t}$ para dos muestras independientes y la prueba $F$ para más de dos grupos independientes. En cuanto al SB, las comparaciones fueron realizados con la inferencia no paramétrica: prueba $\mathrm{U}$ de Mann Whitney para dos muestras independientes y el $\mathrm{H}$ de Kruskal Wallis para más de dos grupos independientes. El análisis estadístico descrito fue realizado en SPSS versión 24 y SPSS AMOS versión 24 , al nivel del $95 \%$ de confiabilidad.

\section{Resultados}

\section{Relación funcional entre Clima Laboral y Síndrome de burnout}

El objetivo principal de la investigación fue conocer la relación funcional entre el CL y el SB en los profesores universitarios, y al realizar la estimación del modelo de medición y el modelo estructural, se encontró que existe relación funcional negativa y significativa entre el $\mathrm{Cl}$ y el SB (coeficiente estandarizado $=-0.374)($ Tabla 3$)$. La relación fue alta entre ambas variables según los indicadores de bondad de ajuste del modelo (AGFI = 0.991) (Tabla 4).

Tabla 3.

Coeficientes del modelo estructural del síndrome de burnout y el clima laboral

\begin{tabular}{|c|c|c|c|c|c|c|c|}
\hline \multicolumn{3}{|c|}{ Relación lineal } & \multirow{2}{*}{$\begin{array}{c}\text { Coeficiente (no } \\
\text { estandarizado) } \\
1.368\end{array}$} & \multirow{2}{*}{$\begin{array}{c}\begin{array}{c}\text { Coeficiente } \\
\text { estandarizado }\end{array} \\
1.257\end{array}$} & \multirow{2}{*}{$\begin{array}{l}\text { S.E. } \\
.161\end{array}$} & \multirow{2}{*}{$\begin{array}{l}\text { C.R. } \\
8.508\end{array}$} & \multirow{2}{*}{$\frac{P}{* * *}$} \\
\hline Supervisión & $<--$ & Clima Laboral & & & & & \\
\hline Burnout & $<--$ & Clima Laboral & -.433 & -0.374 & .093 & -4.663 & $* * *$ \\
\hline Realización Personal & $<--$ & Clima Laboral & 1.000 & 0.912 & & & \\
\hline $\begin{array}{c}\text { Involucramiento } \\
\text { laboral }\end{array}$ & $<--$ & Clima Laboral & 1.432 & 1.465 & .246 & 5.830 & $* * *$ \\
\hline
\end{tabular}




\begin{tabular}{|c|c|c|c|c|c|c|c|}
\hline \multicolumn{3}{|c|}{ Relación lineal } & \multirow{2}{*}{$\begin{array}{c}\text { Coeficiente (no } \\
\text { estandarizado) } \\
.993\end{array}$} & \multirow{2}{*}{$\begin{array}{c}\begin{array}{c}\text { Coeficiente } \\
\text { estandarizado }\end{array} \\
0.930\end{array}$} & \multirow{2}{*}{$\begin{array}{l}\text { S.E. } \\
.050\end{array}$} & \multirow{2}{*}{$\begin{array}{c}\text { C.R. } \\
19.922\end{array}$} & \multirow{2}{*}{$\frac{P}{* * *}$} \\
\hline Comunicación & $<--$ & Clima Laboral & & & & & \\
\hline Condiciones laborales & $<--$ & Clima Laboral & .887 & 0.906 & .043 & 20.663 & $* * *$ \\
\hline Realización Personal & $<--$ & Burnout & 1.000 & 0.730 & & & \\
\hline Despersonalización & $<--$ & Burnout & .573 & 0.755 & .077 & 7.456 & $* * *$ \\
\hline Cansancio Emocional & $<--$ & Burnout & 1.399 & 0.757 & .145 & 9.638 & *** \\
\hline Despersonalización & $<--$ & Supervisión & .169 & 0.210 & .055 & 3.073 & .002 \\
\hline
\end{tabular}

\section{Tabla 4.}

Indicadores de bondad de ajuste del modelo estructural del síndrome de burnout y el clima laboral

\begin{tabular}{lccc}
\hline \multicolumn{1}{c}{ Indicadores } & Medida de ajuste & Valor & $\begin{array}{c}\text { Límite } \\
\text { aceptable* }\end{array}$ \\
\hline Índice de ajuste normado & $\mathrm{NFI}$ & 0.987 & $\geq 0.90$ \\
Índice de ajuste comparativo & $\mathrm{GFI}$ & 0.997 & $\geq 0.90$ \\
Índice ajustado de bondad de ajuste & $\mathrm{AGFI}$ & 0.991 & $\geq 0.85$ \\
Índice relativo de ajuste & $\mathrm{RFI}$ & 0.966 & $\geq 0.90$ \\
Raíz cuadrada del error cuadrático medio & $\mathrm{RMR}$ & 0.808 & $\leq 0.10$ \\
\hline
\end{tabular}

* Byrme, B. (2010)

En relación a los factores del $\mathrm{CL}$, se halló que el factor supervisión se encuentra en relación directa con la despersonalización (Coeficiente estandarizado $=0.210$ ). Igualmente, se observa efectos indirectos de covariabilidad entre la realización personal del $\mathrm{CL}$ con el involucramiento laboral y la supervisión; efecto indirecto de covariabilidad entre el involucramiento laboral con la supervisión, comunicación y condiciones laborales; y el efecto indirecto de covariabilidad entre la supervisión con la comunicación y las condiciones laborales (figura 2). La covariabilidad entre factores, indica que hay otros factores ocultos, latentes o no identificados en el modelo de CL propuesto por Palma (2004).

\section{Figura 2.}

Modelo estructural con coeficientes estandarizados. Relación funcional entre el clima laboral y el síndrome de burnout

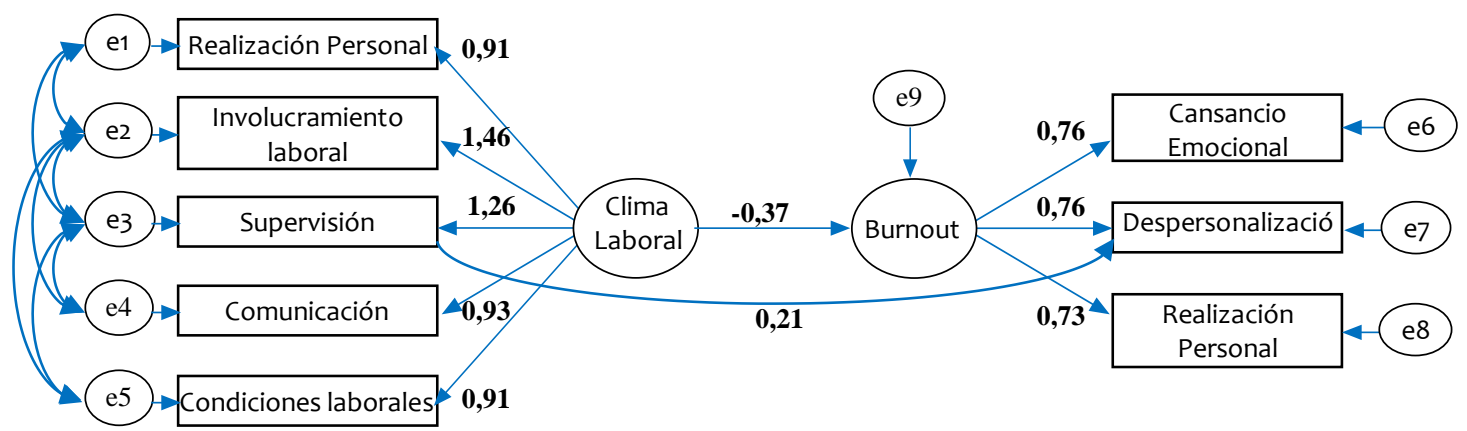

Comparación de los niveles del clima laboral y del Síndrome de burnout, según las variables sociodemográficas-laborales 
De acuerdo al objetivo específico propuesto, referido a comparar los niveles del CL y del SB, según las variables sociodemográficas-laborales, los resultados de la inferencia paramétrica, indicaron que el $\mathrm{CL}$ comparado con las variables referidas, no presentaron diferencias significativas; asimismo los resultados de la inferencia no paramétrica, indicaron que el SB comparado con las variables sociodemográficas-laborales, no presenta diferencias significativas, a excepción de la cantidad de trabajos remunerados, es decir se observa que, a mayor cantidad de trabajos remunerados, mayores son los niveles del SB (Tabla 5).

Tabla 5.

Comparación de los niveles del clima laboral y del síndrome de burnout según las variables sociodemográficas-laborales

\begin{tabular}{lll}
\hline Variables & Clima laboral & Síndrome de burnout \\
\hline Género & Prueba $t: p=0.962$ & U Mann Whitney: $\mathrm{p}=0.355$ \\
Edad & Prueba F: $p=0.277$ & H Kruskal Wallis: $\mathrm{p}=0.221$ \\
Estado civil & Prueba F: $p=0.514$ & H Kruskal Wallis: $\mathrm{p}=0.880$ \\
Condición Laboral & Prueba $t: p=0.127$ & U Mann Whitney: $\mathrm{p}=0.741$ \\
Años de Experiencia & Prueba F: $p=0.071$ & H Kruskal Wallis: $\mathrm{p}=0.933$ \\
Trabajos remunerados & Prueba $\mathrm{t}: \mathrm{p}=0.253$ & U Mann Whitney: $\mathrm{p}=\mathbf{0 . 0 4 0}$ \\
Horas Diarias & Prueba F: $p=0.241$ & H Kruskal Wallis: $\mathrm{p}=0.716$ \\
\hline
\end{tabular}

\section{Discusión}

Según el objetivo general del estudio, los resultados obtenidos evidencian, que existe relación funcional negativa y significativa entre el $\mathrm{CL}$ mediado por sus cinco factores (autorrealización, involucramiento laboral, supervisión, comunicación y condiciones laborales) con el SB modulado por sus tres dimensiones (cansancio emocional, despersonalización y realización personal) (Tabla 3, Tabla 4); es decir a menor CL favorable o muy favorable, mayor será el nivel del SB; por lo tanto, el nivel de CL es un predictor de los niveles del burnout, como lo sostenido por Aronsson et al., (2017). En las organizaciones laborales, cuyo clima laboral es favorable, no existe SB o el SB es de nivel bajo (Moreno y Hidalgo, 2010). Además, el CL favorable o muy favorable sería un factor protector; pero lo inverso sería un factor de riesgo psicosocial para la aparición, mantenimiento o cronicidad del SB.

También los resultados hallados confirman las teorías sobre el CL (Esquer et al, 2019), como los aportes teóricos, que sostienen que el $\mathrm{CL}$, sea como variable interviniente $\mathrm{O}$ independiente, se asocia o tiene efectos sobre variables referentes al comportamiento organizacional y procesos psicológicos como el SB (Palma, 2004); asimismo afecta la satisfacción de los clientes; la calidad de vida en el trabajo, la calidad de los productos o servicios institucionales, la productividad del capital humano y la eficiencia de la acción organizativa (Esquer et al., 2019). Cabe indicar que los resultados obtenidos, es consistente con los hallazgos de estudios precedentes de tipo regional, nacional e internacional, que reportan la relación significativa entre el CL y el SB en muestras de profesores universitarios, (Usán et al., 2018), como en muestras de profesores de educación básica (Farías y Gutiérrez, 2016) y en otros grupos ocupacionales (Mesurado y Laudadío, 2019; Yslado et al., 2020).

Asimismo, el factor de supervisión del CL, está directamente relacionado con la dimensión de despersonalización del SB (Figura 2); lo que indica que el proceso de supervisión no se estaría realizando adecuadamente con un estilo de liderazgo, de apoyo y asesoría adecuados, que 
genere confianza, retroalimentación y mejora continua del desempeño laboral; por lo tanto el profesor universitario ante este proceso presentaría indiferencia, apatía y suspicacia; así como conductas negativas de cinismo, hostilidad, desconfianza y desapego afectivo ante sus colegas, directivos y estudiantes. También, este resultado se explicaría porque el profesor estaría más comprometido e implicado en sus labores de docencia, investigación, responsabilidad social y administrativas y mostraría desinterés por la organización. Al respecto Maslach y Jackson (1997) refieren que los profesores universitarios presentan más sentimientos de despersonalización, que los profesores de primaria y secundaria.

Los resultados hallados indican que existen efectos indirectos de covariabilidad entre varios factores del CL: el factor de autorrealización con los factores de involucramiento laboral y supervisión; el involucramiento laboral con supervisión comunicación y las condiciones laborales (Figura 2); lo que implicaría la existencia de otros indicadores ocultos indirectos o latentes, que no se ajustan a la teoría y modelo del CL de Palma (2004), que sustentan la Escala de clima laboral de SPA, cuya validez y fiabilidad fue determinada con empleados de organizaciones de servicios y producción de Lima Metropolitana. Este instrumento estaría obviando otros factores del CL propuestos por expertos (Vela, Garrido y Pérez, 2016); o incluye una dimensión no organizacional, como la autorrealización que está relacionada con la personalidad y genera disturbio en las covarianzas, que fue encontrado en el estudio de Salazar y Serpa (2017), o la muestra estudiada percibe indicadores latentes, propios de un contexto universitario público de una provincia del Perú.

Con respecto a los niveles del CL (Tabla 1); se halló que el $\mathrm{CL}$ es promedio (ni favorable, ni desfavorable), y también el burnout es de nivel promedio en los profesores universitarios investigados; es decir el CL promedio favorece el no incremento del SB y sus dimensiones. La mayoría de profesores percibe un entorno laboral relativamente adecuado, en el cual se labora sin mayores presiones, ni cambios en el estilo de gestión administrativa; lo cual tampoco es el óptimo para el desarrollo institucional, ni lograr la competitividad y el aseguramiento de la calidad en la prestación de servicios educativos, aspectos que exigen las políticas públicas internacionales para la educación superior universitaria (Organización de las Naciones Unidas para la Educación, la Ciencia y la Cultura [UNESCO], 2017), y son necesarios para el licenciamiento y acreditación continua de las universidades públicas peruanas (SINEACE, 2017).

Un resultado similar al descrito en el párrafo anterior fue reportado por Palma (2004), que concluye que el personal administrativo y docente de tres universidades privadas de Perú, perciben un clima laboral promedio. Resultados diferentes se reportaron en otros estudios latinoamericanos; así en las universidades de Sucre (Colombia), se halló que el clima laboral fue inadecuado, porque las condiciones laborales no son las mejores, pues existe inestabilidad laboral, y apoyo insuficiente para el crecimiento profesional y autorrealización, que generan desmotivación e inciden en el desempeño laboral, la calidad del servicio y bienestar de los profesores. Acotando al respecto, se sostiene que un clima laboral favorable y positivo, tiene efecto en la satisfacción laboral intrínseca y en la menor intención de rotación de los profesores universitarios (Patlán y Martínez, 2017).

Referente a los niveles del SB (Tabla 2); se encontró que en promedio los profesores presentan burnout de nivel medio, igualmente las tres dimensiones del burnout son de nivel medio; sin embargo, una tercera parte ya presenta SB alto y se encuentra en riesgo, requiere de una atención especializada y una intervención ocupacional a corto plazo, para reducir el SB. La tendencia del incremento del SB medio y alto en el colectivo profesional estudiado es mundial, según lo reportado en estudios precedentes realizados en el siglo XXI (Arquero y Donoso, 2006, 2013; Bayona y Jaller, 2016; Carpio y Requis, 2016; Ilaja y Reyes, 2016; Jiménez, Arrieta y Quintero, 2017; Lou y Chen, 2016; Quispe, 2017; Rivera, Segarra y Giler, 2018); en 
menor frecuencia otros estudios realizados en profesores de universidades latinoamericanas, reportaron lo contrario, hallaron burnout de nivel bajo (Palmer et al., 2016; Mesurado y Laudadío, 2019). Se acota que el SB alto es una variable predictora de renuncia del personal (Córdova, 2018), cuyas consecuencias negativas afectan simultáneamente al profesor universitario, a la universidad y al entorno social.

De acuerdo al objetivo específico del estudio, los resultados encontrados en este estudio revelan que no existen diferencias significativas al comparar los niveles del $C L$, según las variables sociodemográficas-laborales de: sexo, edad, estado civil, condición laboral, tiempo de experiencia laboral, número de trabajos remunerados, horas diarias laboradas en contacto con los estudiantes (Tabla 5); lo que implica, que el nivel de clima de trabajo promedio, en general es percibido indistintamente por lo profesores, cual sea su perfil sociodemográfico y laboral. Este resultado es similar al hallado por Palma (2004), que reportó que no existen diferencias en la percepción del clima laboral de los profesores universitarios, cuando se comparan sus promedios según sexo, grupo ocupacional y tiempo de servicio. Entonces las variables sociodemográficas y laborales estudiadas no son predictoras de los niveles de CL.

Asimismo, al comparar los niveles del SB, de acuerdo con las variables sociodemográficas y laborales señaladas (Tabla 5), se encontró que no existe diferencias significativas, a excepción de la cantidad de trabajos remunerados, lo que significa que, a mayor cantidad de trabajos remunerados, mayores son los niveles de SB. De la revisión de la literatura se infiere, que no hay consenso sobre el tema en cuestión, quizás en parte por los diferentes puntos de corte utilizados para establecer los niveles del SB; sin embargo algunos estudios precedentes informaron resultados similares (Lou y Chen, 2016), y en otros, se dieron a conocer que las variables de sexo, edad, número de horas laboradas con alumnos, problemas de salud, tiempo dedicado a la investigación, tiempo de experiencia y condición laboral, entre otros estaban asociados al SB (Arquero y Donoso, 2013; González et al., 2015; Rivera et al., 2018).

Los nuevos conocimientos y aplicaciones prácticas derivadas de los resultados de este estudio son válidos, confiables y generalizables a la población de estudio. Los aportes prácticos son: a) La elaboración de una aplicación para web, orientada a la administración, calificación e interpretación individual o colectiva de los protocolos de los instrumentos de medición del $C L$ y del SB, para fines de investigación y medir el $C L$ y SB periódicamente para la toma de decisiones sobre las prácticas, políticas, estructuras, procesos y sistemas del CL, que inciden en los comportamientos, actitudes, motivación de los profesores, para mejorar la calidad de vida laboral, salud ocupacional, satisfacción laboral y productividad de los trabajadores (De Araujo et al., 2019). b) En base a los resultados se puede implementar un programa de intervención ocupacional, con el fin de reducir el SB y un programa de intervención organizacional, para optimizar el CL, y así mejorar la percepción desfavorable de los factores de supervisión, las condiciones laborales y comunicación, que son variables que influyen en la prevalencia e incidencia del SB en los profesores (Tabares-Diaz et al., 2020). Además, es necesario reforzar la percepción promedio del involucramiento laboral y autorrealización, que son factores relacionados con los procesos psicológicos (moral, afectividad, motivación y personalidad) de los profesores, e implementar la asignación de bonos emocionales de reconocimiento y revalorización de los profesores, que constituyen un potencial para promover el cambio organizacional.

Las limitaciones del estudio fueron: a) Se utilizó un diseño transversal; por lo tanto, los resultados encontrados no establecen relaciones de causa-efecto, y son válidos temporalmente. b) El número de participantes se controló solo por facultades (afijación proporcional). c) No todos los profesores aceptaron participar en el estudio con consentimiento informado escrito; por lo tanto, hubo valores perdidos; pero como la 
población fue amplia se reemplazaron aleatoriamente para completar la muestra. d) La muestra ha sido obtenida de una única universidad de Perú; pero es representativa de la universidad pública típica de Perú y como tal los resultados pueden ser ampliables a cualquier universidad similar en Perú y en futuros estudios de investigación se sugiere replicar el estudio con muestras más amplias de profesores de otras facultades y/o nacionalidades. Además se debe realizar investigaciones sobre la actualización de las propiedades psicométricas de la Escala de clima laboral de Palma (2004), para que este instrumento evalúe el clima laboral apropiadamente en el contexto de las universidades (cuyo modelo de estructura y diseño organizacional es diferente a las empresas de servicios y producción), que permita identificar no solo el clima laboral básico, sino distintos tipos de clima laboral relacionados con la baja y alta efectividad de la organización, como lo sugerido por Ramos y Tejera (2017). También es pertinente realizar el análisis confirmatorio del Cuestionario de "burnout" para profesores universitarios, para su validación en el contexto universitario nacional e internacional.

\section{Conclusiones}

Existe evidencia para afirmar que el $\mathrm{CL}$ y sus cinco factores se relaciona negativa y significativamente con el SB y sus tres dimensiones, en los profesores de una universidad pública de Perú. El CL comparado según las variables sociodemográficas-laborales de estado civil, sexo, edad, condición laboral, tiempo de experiencia laboral, horas diarias laboradas en contacto con los estudiantes, número de trabajos remunerados, no presenta diferencias significativas. El SB comparado según las variables sociodemográficas-laborales citadas, no presenta diferencias significativas; a excepción de la cantidad de trabajos remunerados, se observa que, a mayor cantidad de trabajos remunerados, los niveles de SB son más altos.

\section{Referencias}

Abkhou, T., \& Jenaabadi, H. (2015). Comparative Analysis of the Relationship between Job Burnout and General Health of Male and Female High School Teachers in Zabol. Natural Science, 07(08), 391-399. https://doi.org/10.4236/ns.2015.78042

Arís, N. (2017). El Síndrome de Burnout en los docentes. Electronic Journal of Research in Education Psychology, 7(18). https://doi.org/10.25115/ejrep.v7i18.1324

Aronsson, G., Theorell, T., Grape, T., Hammarström, A., Hogstedt, C., Marteinsdottir, I., ... \& Hall, C. (2017). A systematic review including meta-analysis of work environment and burnout symptoms. BMC public health, 17(1), 1-13. https://doi.org/10.1186/s12889-0174153-7

Arquero, J. \& Donoso, J. (2006). Instrumento de medición del síndrome de quemarse en el trabajo (burnout) en profesores universitarios. Publicación Contabilidad y Auditoría. 23 - Año 12. https://ojs.econ.uba.ar/index.php/Contyaudit/article/view/110

Arquero, J. \& Donoso, J. (2013). Docencia, investigación y burnout: el síndrome del quemado en profesores universitarios de Contabilidad. Revista de Contabilidad. 16(2), 94-105. https://doi.org/10.1016/j.rcsar.2013.04.001

Bayona, S. \& Jaller, C. (2016). Prevalencia y factores asociados a síndrome Burnout en docentes vs personal administrativo pertenecientes a una universidad privada en Bogotá. (Tesis de Especialización en Epidemiología). Universidad del Rosario. Colombia. Recuperado de http://repository.urosario.edu.co/handle/10336/12599?show=full 
Cárdenas, M., Méndez, L. M., \& González, M. T. (2014). Evaluación del desempeño docente, estrés y burnout en profesores universitarios / Evaluation of teacher performance, stress and burnout in university professors. Actualidades Investigativas en Educación, 14(1). https://doi.org/10.15517/aie.v1411.13210

Carpio, G. \& Requis, S. (2016). Nivel de Síndrome de Burnout en enfermeras docentes de práctica que laboran en una Universidad Privada de Lima. Ágora Revista Científica, 03(01), 329-336. https://doi.org/10.21679/arc. v3i1.60

Collier, J. E. (2020). Applied structural equation modeling using AMOS: Basic to advanced techniques. Routledge.

Córdova, C. (2018). Retención del talento humano: variables predictoras de renuncia e instrumentos de medición. En E. Louffat (Ed.). Indicadores y mediciones aplicados a la gestión de personas. Un análisis desde diversas perspectivas (pp. 362-403). Perú: Pearson.

De Araujo, T. I., Costa, J. P., De Costa, F. L. , Fernandes, X. D. , Medeiros, D. M., \& Souza, E. E. (2019). Prevalência e fatores associados da síndrome de Burnout em docentes universitários. Revista Brasileira de Medicina do Trabalho, 17(2), 170-179. https://doi.org/10.5327/Z1679443520190385

Espinoza, I. M., Tous-Pallarès, J., \& Vigil-Colet, A. (2015). Efecto del clima psicosocial del grupo y de la personalidad en el síndrome de quemado en el trabajo en docentes. Anales de Psicología, 31(2), 651. https://doi.org/10.6018/analesps.31.2.174371

Esquer, L. A. Z., Ruíz, H. D. M., Guerrero, J. C., Ambrosio, O. V., de Nava Tapia, S. L., Pacheco, C. B., \& Lirios, C. G. (2019). Composición factorial exploratoria del clima laboral. Hologramática, 30(1), 29-43. Recuperado de https://www.cienciared.com.ar/ra/doc.php?n=2172

González, G., Carrasquilla, D., Latorre de la Rosa, G., Torres, V., \& Villamil, K. (2015). Síndrome de Burnout en docentes universitarios. Revista cubana de enfermería, 31(4). Recuperado de http://www.revenfermeria.sld.cu/index.php/enf/article/view/902/138

Hernández, C. E., \& Carpio, N. (2019). Introducción a los tipos de muestreo. Alerta, Revista científica del Instituto Nacional de Salud, 2(1), 75-79. https://doi.org/10.5377/alerta.v2i1.7535

Hernández, H., Garrido, N., \& Rico, C. (2016). Diseño de instrumento para evaluar clima organizacional de universidades públicas en México. European Scientific Journal, 12 (28), 312-328. 12 http://dx.doi.org/10.19044/esj.2016.v12n28p312

Ilaja, B. \& Reyes, C. (2016). Burnout y estrategias de inteligencia emocional en profesores universitarios: implicaciones en la salud laboral educativa. Psicología desde el caribe, 33(1), 31-46. https://doi.org/10.14482/ psdc.33.1.8081

Jiménez, L., Arrieta, M. \& Quintero, K. (2017). Síndrome de Burnout y evaluación de desempeño en docentes de la Fundación Universitaria del área andina sede Valledupar. Escenarios, 15(1), 36-46. https://doi.org/10.15665/esc.v15i1.1120

Lou, Y. G., \& Chen, L. Y. (2016). A Study of the English Teachers' Burnout in a Local Comprehensive University in China. Creative Education, 7, 646-654. https://doi.org/10.4236/ce.2016.74067

Maslach, C. \& Jackson, S. (1997). MBI Inventario "Burnout" de Maslach. Síndrome del quemado por estrés laboral asistencial. Madrid: TEA Ediciones, S.A. 
Mazzanti, M. A. (2011). Declaración de Helsinki, principios y valores bioéticos en juego en la investigación médica con seres humanos. Revista Colombiana de Bioética, 6(1), 125144. https://doi.org/10.18270/rcb.v6i1.821

Medrano, L. A. \& Muñoz-Navarro, R. (2017). Aproximación Conceptual y Práctica a los Modelos de Ecuaciones Estructurales. Revista Digital de Investigación en Docencia Universitaria, 11(1), 219-239. https://doi.org/10.19083/ridu.11.486

Morales, L. Y. P., Vélez, R. P., Ramírez, C. M., Largada, M. F., Martínez, R. L., \& Rodríguez, G. R. (2016). Prevalencia del Síndrome de Burnout en docentes de la Universidad Autónoma de Baja California, Mexicali, México. Revista Cubana de Salud y Trabajo, 17(3), 36-40. Recuperado de http://www.bvs.sld.cu/revistas/rst/vol17_3_16/rst07316.pdf

Moreno, C.A., \& Hidalgo, P.M. (2011). Relación entre el clima organizacional y el síndrome de burnout en empleados de una firma de vigilancia en una empresa carbonera de Guajira (Colombia). Revista Psicogente, 13(24), 1-20. Recuperado de http://revistas.unisimon.edu.co/index.php/psicogente/article/view/1795

Mesurado, B., \& Laudadío, J. (2019). Experiencia profesional, capital psicológico y engagement. Su relación con el burnout en docentes universitarios. Propósitos y Representaciones, 7(3), 12 - 40. http://dx.doi.org/10.20511/pyr2019.v7n3.327

Palma, S. (2004). Escala clima laboral CL - SPC. Lima, Perú: CARTOLAN E.I.R.L.

Palmer, L., Prince, R., Medina, C., Figueroa, M., López, R. \& Rodríguez, G. (2016). Prevalencia del síndrome de burnout en docentes de la Universidad Autónoma de Baja California, Mexicali, México. Revista Cubana de Salud y Trabajo, 17(3), 36-40. Recuperado de http://www.revsaludtrabajo.sld.cu/index.php/revsyt/article/view/54

Patlán, J. \& Martínez, E. (2017). Evaluación de la imagen organizacional universitaria en una institución de educación superior. Contaduría y Administración. 62(1), 105-122. https://doi.org/10.1016/j.cya.2016.07.002

Quispe, Y. (2017). Síndrome de burnout en docentes de la escuela profesional de administración. Revista de Investigaciones de la Escuela de Posgrado-UNA, 6(1), 11-18. https://doi.org/10.26788/epg.v6i1.3

Ramirez, E. H., Espinoza, M. R., Esquivel, S. M. \& Naranjo, M. E. (2020). Inteligencia emocional, competencias y desempeño del docente universitario: Aplicando la técnica mínimos cuadrados parciales SEM-PLS. Revista Electrónica Interuniversitaria de Formación del Profesorado, 23(3). 99-114. https://doi.org/10.6018/reifop.428261

Ramos, V., \& Tejera, E. (2017). Estudio de relaciones entre cultura, clima y fuerza de clima laboral en Ecuador. Acción psicológica, 14(2), 225-239. https://doi.org/10.5944/ap.14.2.17046

Rivera, A., Segarra, P., \& Giler, G. (2018). Síndrome de burnout en docentes de instituciones de educación superior. Archivos Venezolanos de Farmacología y Terapéutica, Caracas, 37(2), 17-23. Recuperado de http://www.revistaavft.com/images/revistas/2018/avft_2_2018/numero_2_2018.html

Rodríguez-García, A.M., Sola-Martínez, T. \& Fernández-Cruz, M. (2017). Impacto del Burnout en el desarrollo profesional del profesorado universitario. Una revisión de la investigación. Revista Electrónica Interuniversitaria de Formación del Profesorado, 20 (3), 161-178. http://dx.doi.org/10.6018/reifop.20.3.275121 
Ruíz de Chávez, D., Pando, M., Aranda, C., \& Almeida, C. (2014). Burnout and Work Engagement in Zacatecas University Teachers. Ciencia \& Trabajo, 16(50), 115-120. https://doi.org/10.4067/S0718-24492014000200010

Salazar, C., \& Serpa, A. (2017) Análisis confirmatorio y coeficiente Omega como propiedades psicométricas del instrumento Clima Laboral de Sonia Palma. Revista de Investigación en Psicología, 20(2), 377-388. https://doi.org/10.15381/ rinvp.v20i2.14047

SINEACE (2017). Modelo de acreditación institucional para universidades. Sistema Nacional de Evaluación, Acreditación y Certificación de la Calidad Educativa, Perú. Recuperado de https://www.sineace.gob.pe/wp-content/uploads/2017/07/Anexo-Resoluci\%C3\% B3n-N\%C2\%B0279-2017.pdf

Tabares-Díaz, Y. A., Martínez-Daza, V. A., \& Matabanchoy-Tulcán, S. M. (2020). Síndrome de Burnout en docentes de Latinoamérica: Una revisión sistemática. Universidad y Salud, 22(3), 265-279. https://doi.org/10.22267/rus.202203.199

UNESCO, (2017). Documento de política 30. Seis maneras de asegurar que la educación superior no deje a nadie atrás. Organización de las Naciones Unidas para la Educación, la Ciencia y la Cultura Recuperado de http://unesdoc.unesco.org/images/0024/002478/247862S.pdf

Usán, P., Salavera, C., \& Domper, E. (2018). ¿Cómo se interrelacionan las variables de burnout, engagement y autoeficacia académica? Un estudio con adolescentes escolares. Revista Electrónica Interuniversitaria De Formación Del Profesorado, 21(2), 141-153. https://doi.org/10.6018/reifop.21.2.311361

Vela, H. Garrido, N., \& Pérez C. (2016). Diseño de Instrumento para Evaluar Clima Organizacional de Universidades Públicas en México. European Scientific Journal 12(28), 312. https://doi.org/10.19044/esj. 2016.v12n28p312

Yslado, R. M., Ramírez, E. H. \& Espinoza, M. R. (2020). Burnout, docencia e investigación en profesores universitarios de la Facultad de Ciencias Empresariales de Perú y España. Educade Revista de Educación en Contabilidad, Finanzas y Administración de Empresas, (11), 3-19. http://dx.doi.org/10.12795/EDUCADE.2020.111.02 